\title{
Streamlining the Design Tradespace for Earth Imaging Constellations
}

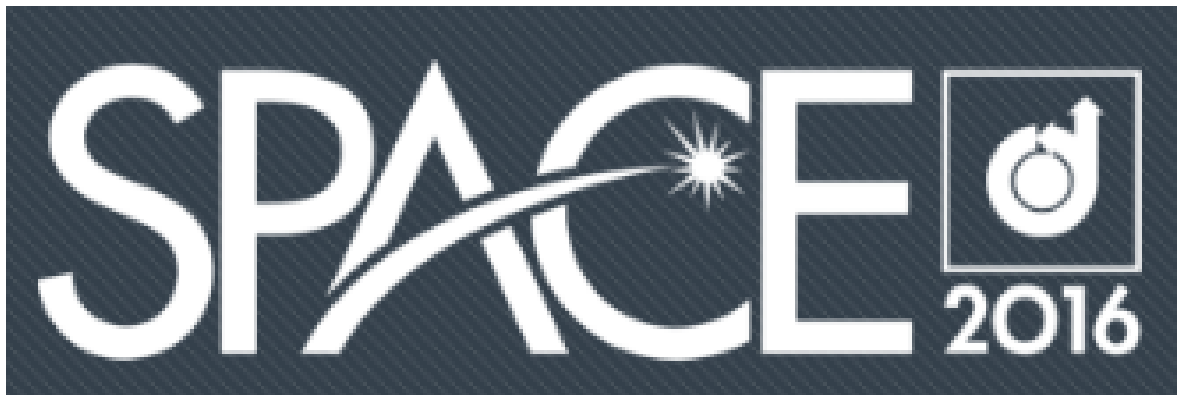

Sreeja Nag ${ }^{1,2}$, Steven P. Hughes ${ }^{1}$ and Jacqueline J. Le Moigne ${ }^{1}$

${ }^{1}$ NASA Goddard Space Flight Center

${ }^{2}$ Bay Area Environmental Research Institute 


\section{Tradespace Design Context}

\section{Distributed Spacecraft Missions}

Tradespace exploration is required early in the design cycle

Performance: Improve sampling in spatial (synthetic apertures), temporal (constellations), spectral (fractionated $\mathrm{S} / \mathrm{C}$ ), angular (formations) dimensions

Cost: Need more inter-operability planning, autonomy, scheduling commands + data, ground station networks

Ilities in Operations: Flexibility, Reconfigurability, Scalability, etc.

Better Design: Many conflicting variables and objectives thus better methods needed in Phase At - coupled models, machine learning, planning/scheduling methods, etc.

NASA GSFC is building a software tool called Tradespace Analysis Tool for Constellations (TAT-C), to address some of the above questions.
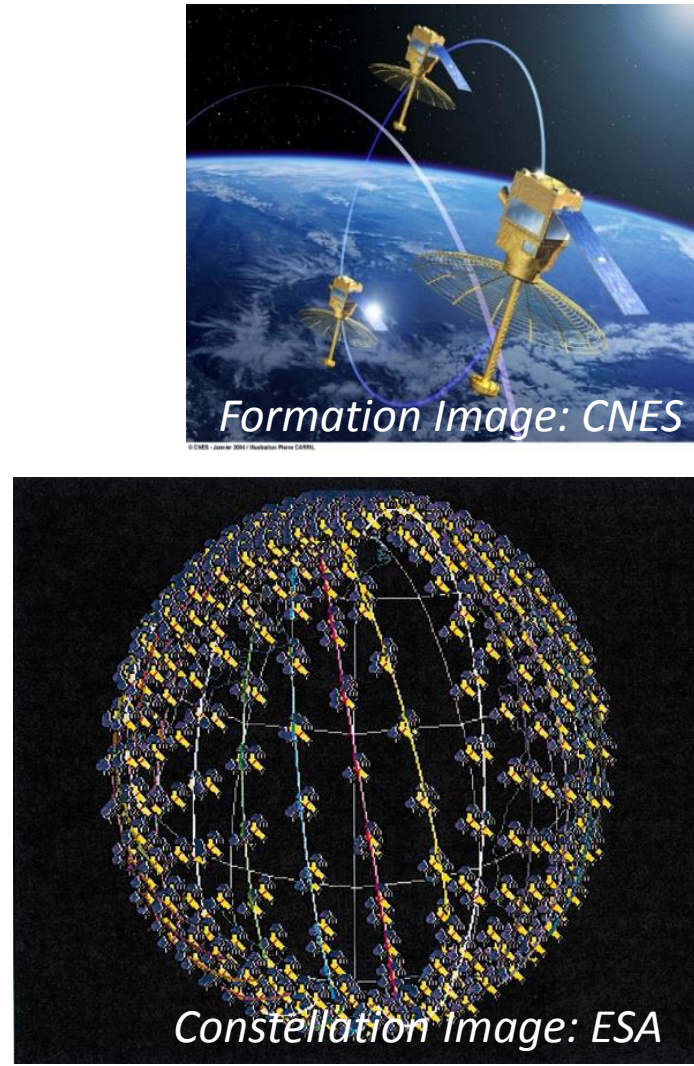

Fractionated S/C Image: DARPA

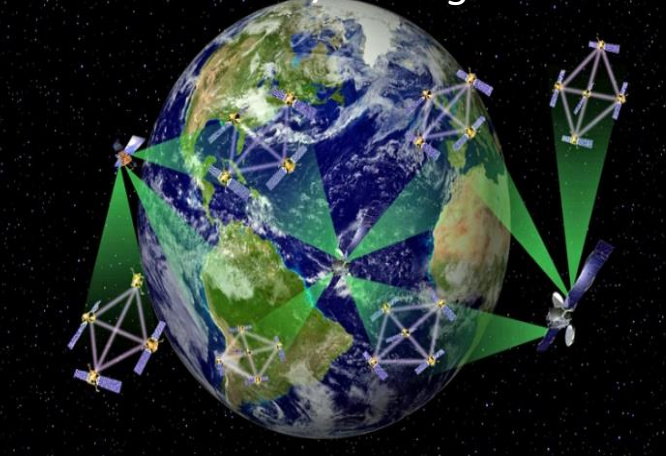




\section{Tradespace Search Iterator}

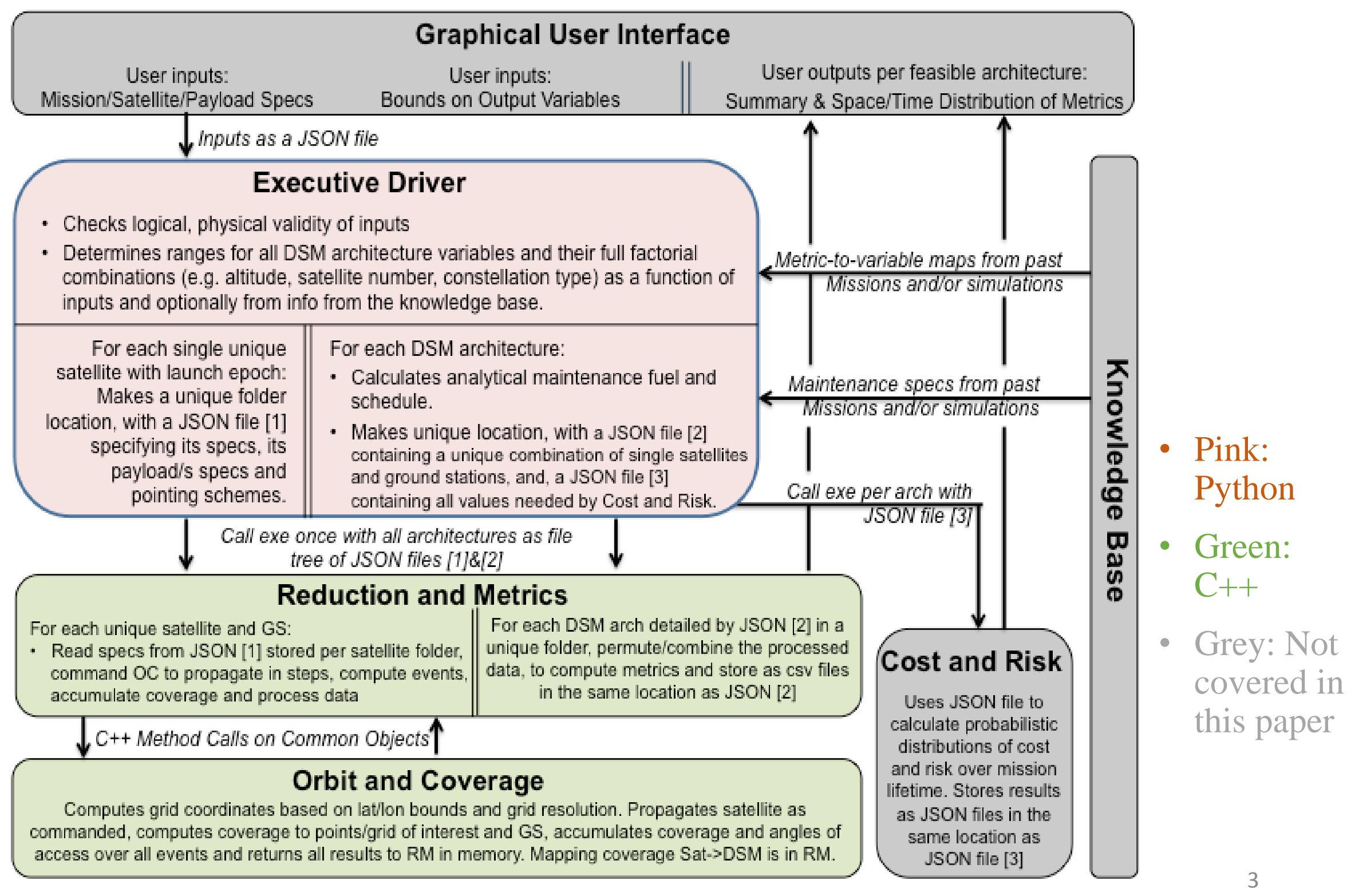


Green highlighted rows correspond to variables needed only for pairs missions, including occultations with satellite pair option.

Blue higlighted rows correspond to variables needed only for occultation missions. Yellow highlighted rows correspond to variables needed only for imaging missions.

\section{Inputs}

to the

Exec

Driver:

- Yellow:

Imaging

- Green: Pairs

of sats

- Blue: Occultation

\begin{tabular}{|c|c|c|}
\hline \multicolumn{3}{|r|}{ Mission Loncept } \\
\hline Attribute & Characteristics & Description \\
\hline Mission Dwner & Select & Select from 'Academic', 'Military', 'Commercial', or'Government'. \\
\hline Start Epoch & UTC time & Date when the satellites will initialize. Default is current time. \\
\hline Mission Duration & exact & Total time the mission is expected to last, starting from the first launch. Default is 30 days. \\
\hline Performance Period & min,max & Ranges of times within mission duration, when outputs will be calculated. Default is the first 30 days. \\
\hline Area of Interest & exact locations & Lat'Lon'Ált list or Lat'Lon bounds. Global is default. \\
\hline Ground Stn options & Select andior file & If the user has existing satellites to complement. Row=GS num, columns=GS lat, lon, alt, band. Default is NEN. \\
\hline Launch preferences & Select andior file & If the user has existing satellites to complement. Row=LV num, columns=LV specs. Default is all. \\
\hline Propagation fidelity & low, med, high & Three levels of propagators to be selected against (time and description provided). Default is low. \\
\hline Output options & Select & Which of the output veriables is the user interested in. Default is all but angles. \\
\hline Qutput bounds & min, mak & Min and Mas for any of the variables in the Dutputs sheet. Default is in the output sheet. \\
\hline
\end{tabular}

\begin{tabular}{|c|c|c|}
\hline \multicolumn{3}{|r|}{ Orbit Specifications } \\
\hline Attribute & Characteristics & Description \\
\hline Existing Sat options & Select andior file & User has a saticonstellation in mind. Default is none. \\
\hline Number of new Sats & min, max & Number of new sats allowed in the DSM in addition to existing. One and ten is default respectively. \\
\hline Altitude Range of Interest & Select or min, max & Ranges of altitudes the user is interested in, LEQ $(300-1000 \mathrm{~km})$ is def ault. \\
\hline Inclination Range of Interest & $\min , \max$ & Ranges of inclinations the user is interested in, 50-90 deg is default. \\
\hline Special Drbits only & Select & Select between only SSO with LT option, frozen, critically inclined, ISS \\
\hline Number of satellite types & exact & Enter 1 if const. is homogeneous or number, if heterogeneous. Whetero, allsatelhe specs per instance. \\
\hline
\end{tabular}

\begin{tabular}{|c|c|c|}
\hline \multicolumn{3}{|r|}{ Satellite Specifications } \\
\hline Attribute & Characteristics & Description \\
\hline Satellite Mass & Approximate & Default is $2200 \mathrm{~kg}$. \\
\hline Satellite Volume & Approsimate & Default is $1550 \mathrm{~W}$. \\
\hline Satellite Power & Approsimate & Default is $26.4773 \mathrm{~m} 3$ \\
\hline Maximum Slew Angle & min, max & Maximum coning angle in the along and cross track for a scanning satellite. Default is 0 . \\
\hline SoanRate & min, max & Scan rate of the satellite, betw een the maximum angles. Default is 0. \\
\hline Number of payloadsisat & Exact & For multi-instrument sats. Default is one. If more than one, al/ paghadefspess tperinstanee. \\
\hline Commband & exactbands & For downlinking data to ground stations. Default is Ka-band. \\
\hline \multicolumn{3}{|r|}{ Payload Specifications } \\
\hline Attribute & Characteristics & Description \\
\hline Doculation or Imaging or Pairs & Select & Determines payload's measurement conops. Imaging is default. \\
\hline Payload Mass & Approximate & Default is $420 \mathrm{~kg}$. Payload mass will be checked within reasonableness of sat mass. \\
\hline Payload Volume & Approximate & Default is 590 W. Payload volume will be checked within reasonableness of sat volume. \\
\hline Payload Power & Approximate & Default is $4 \mathrm{~m} 3$. Payload power will be checked within reasonableness of sat power. \\
\hline Payload TRL & exact & Where is the payload at in the development cycle? \\
\hline Radiometric resolution & $\min$ & Number of bits per pikel. 12 bits is default. \\
\hline Dcoultation or Pairs coupling & Exact or file & Mark observer or occulterlpair, for every occulting or pairs mission. \\
\hline $\begin{array}{l}\text { Nadir swath or FOV } \\
\text { Nadir GSD or iFOU }\end{array}$ & min, max $\mathrm{QR}$ exact & Conical or rectangular dimensions of the full spot size. Default is 15 deg in Along and Cross track. \\
\hline Object's of interest & Select andior file & Select between celestial body or 'satellite' if an occultation mission. Fill row 33 if latter. Default is Sun. \\
\hline Dcoultation Altitude & max, min & Tangent altitudes between which occultation measurements will be made. Default is $10-50 \mathrm{~km}$. \\
\hline Measurement time & min, max & Sum of exposure and integration time per image or measurement. Default is half-pixel travel time. \\
\hline Solar conditions & Select & Determines if the sat is sunlit or eclipsed or agnostic when measuring. Default is agnostic. \\
\hline Sun Glint preference & Select & Select if sun glint ( $<5$ deg relative Azimuth) to be included, avoided or no preference. Default is no preference. \\
\hline Spectral or other Channels & Exact wavelengths & Central wavelength for multi-spectral imaging. $300: 100: 1000 \mathrm{~nm}$ is default. \\
\hline Spectral resolution & exact binwidths & Band or bin width of each central w avelength in the spectral range. $50 \mathrm{~nm}$ is default. \\
\hline
\end{tabular}




\section{Outputs from the RM Module}

All the listed outputs except * will be available per architecture, per ground spot, per unit time. The characteristics indicate bounds that the user can set as inputs.

Output tabs will include panels for attributes vs. cost, spatial bins and time series.

\begin{tabular}{|c|c|c|}
\hline \multicolumn{3}{|r|}{ Spatial Metrics } \\
\hline Attribute & Characteristics & Description \\
\hline Effective Spatial Resolution & min, max, average & Ground pixel size. \\
\hline Effective Swath & min, max, average & Cross and along track extent of one full image. \\
\hline Percentage Image overlap & min, max, average & $\%$ of every image that overlaps with another. $100 \%$ for complete 2 -fold and $0 \%$ for none. \\
\hline Covered positions (w/ FOV) & lat, lon & Spatial positions where imaging measurements are made per sat per arch within I/P "Area of Interest". \\
\hline Percentage POI covered & $\min , \max$ & Percentage of the required points of interest within the area of interest covered within mission performance period. \\
\hline Spacecraft Ephemeris & lat, lon, alt & Ephemeris over time for all constituent spacecraft in a DSM. \\
\hline Occultation positions & lat, lon & Spatial positions where occultation measurements are made per sat per arch within I/P "Area of Interest". \\
\hline Inter-Sat Range and Rate & min, max, average & Distances and Rate (AT,CT,R,euclid) between each satellite in the virtual group (stereo) \\
\hline Possible positions (w/ FOR) & lat, lon & Spatial positions where imaging measurements CAN BE made per sat per arch within I/P "Area of Interest". \\
\hline
\end{tabular}

- Yellow: Imaging

- Green: Pairs of sats

- Blue: Occultation

\begin{tabular}{|c|c|c|}
\hline \multicolumn{3}{|r|}{ Temporal Metrics } \\
\hline Attribute & Characteristics & Description \\
\hline Occultation time* & min, max, average & If an occult mission, time which each occultation lasts for \\
\hline$\%$ period time in Sun* & $\min$, max, average & Fraction of the orbit that the sat spends in the Sun (vs. eclipsed) \\
\hline Time to Coverage* & $\min$, max, average & Time to cover the "Area of Interest" entirely once \\
\hline Access Time* & $\min$, max, average & Time that any ground spot has access to a satellite (within FOR) \\
\hline Latency to downlink* & $\min$, max, average & Time between observation and downlink to the next ground station \\
\hline Repeat Time* & $\min$, max, average & Time between repeats (within 1 deg of view angle) of every point in the "Area of Interest". Calc. for virtual and real sats for Stereo/Comm missions \\
\hline Revisit Time* & $\min$, max, average & Time between revisits of every point in the "Area of Interest" \\
\hline
\end{tabular}

\begin{tabular}{|lll|}
\hline & & \\
\hline & Characteristics & Description \\
\hline Attribute & $\min , \max$ & Between the payload-target vector and zenith, if imaging mission. Default is none for all angles. \\
View Zenith Angle & $\min , \max$ & Between the payload-target vector projection on target normal plane and true north projection on the same plane, , if imaging mission \\
View Azimith Angle & $\min , \max$ & Between the sun-target vector and zenith for day measurements, if imaging mission \\
Solar Zenith Angle & $\min , \max$ & Between the sun-target vector projection on target normal plane and true north projection on the same plane, if imaging mission \\
Solar Azimuth Angle & $\min , \max$, average & For night measurements. \\
Lunar phase &
\end{tabular}

\begin{tabular}{|lll|}
\hline \multicolumn{2}{|c|}{ Radiometric Metrics } \\
\hline Attribute & Characteristics & Description \\
Signal to Noise Ratio & min, average & Expected signal and noise (SNR) of each architecture's satellites with respect to a selected one. \\
\hline
\end{tabular}

Green highlighted rows correspond to variables needed only for pairs missions, including occultations with satellite pair option.

Blue higlighted rows correspond to variables needed only for occultation missions. Yellow highlighted rows correspond to variables needed only for imaging missions. 


\section{and Metric Computation}

\section{Example of a JSON file capturing ED inputs:}

"MissionConcepts" :

"StartEpoch"

"MissionDuration":" 0:2592000"

"PerformancePeriod": : "0:2592000"

"AreaofInterest" : "Landsat_landImages.txt"

"ObjectsofInterest" : " "

"GroundStationoptions": "DSN"

"LaunchPreferences" : "Primary",

"MissionDirector" : "Government"

"Satelliteorbits":

"ExistingSatelliteoptions" : " ",

"NumberofNewSatellites": "1:8"

"AltitudeRangesofInterest" : "710:710"

"InclinationRangesofInterest": "98.2:98.2"

"Specialorbits"

" "

"ObservatorySpecifications": "ObservatorySpecifications.txt", "InstrumentSpecifications" : "InstrumentSpecifications.txt"

"OutputBounds" : f

"тіmeтоCoverage"

"Accesstime"

"RevisitTime"

"Crossoverlap"

"Alongoverlap"

"SignalNoiseRatio" :

"LunarPhase"

"Obszenith"

"ObsAzimuth"

"Sunzenith"

"DownlinkIatency" :

"SunAzimuth"

"SpatialResolution" :

"CrossSwath"

"Alongswath"

"ObsLatitude"

"ObsLongitude"

"Obsaltitude"

"Objzenith"

"ObjAzimuth"
Example of a file tree capturing ED outputs:

User Directory

-TradespaceSearchRequest.json InstrumentSpecifications.txt ObservatorySpecifications.txt Landsat_landImages.txt Mono

—Orb0000_GS00_Pnt000_Pay00

-ReductionMetrics.json ephemeris.txt angles.txt

-Orb0001_GS00_Pnt000_Pay00

-Orb0002_GS00_Pnt000_Pay00

Orb0003_GS00_Pnt000_Pay00

-DSMs

-Subspace00000

-Manifest.json CostRisk.json gbl.csv lcl.csv CostOutput.json Subspace00001 Subspace00002 


\section{Preliminary Sizing for Streamlining}

\section{Spatial Metric Dependence on Constellation Design Variables:}

Given altitude, maximum spatial resolution or ground sample distance (GSD) is limited by maximum FOV. Even w/ awide angle FOV, only those pixels in the retrieved image that satisfy max GSD will be useful. Thus, the effective FOV is limited by the GSD limits.

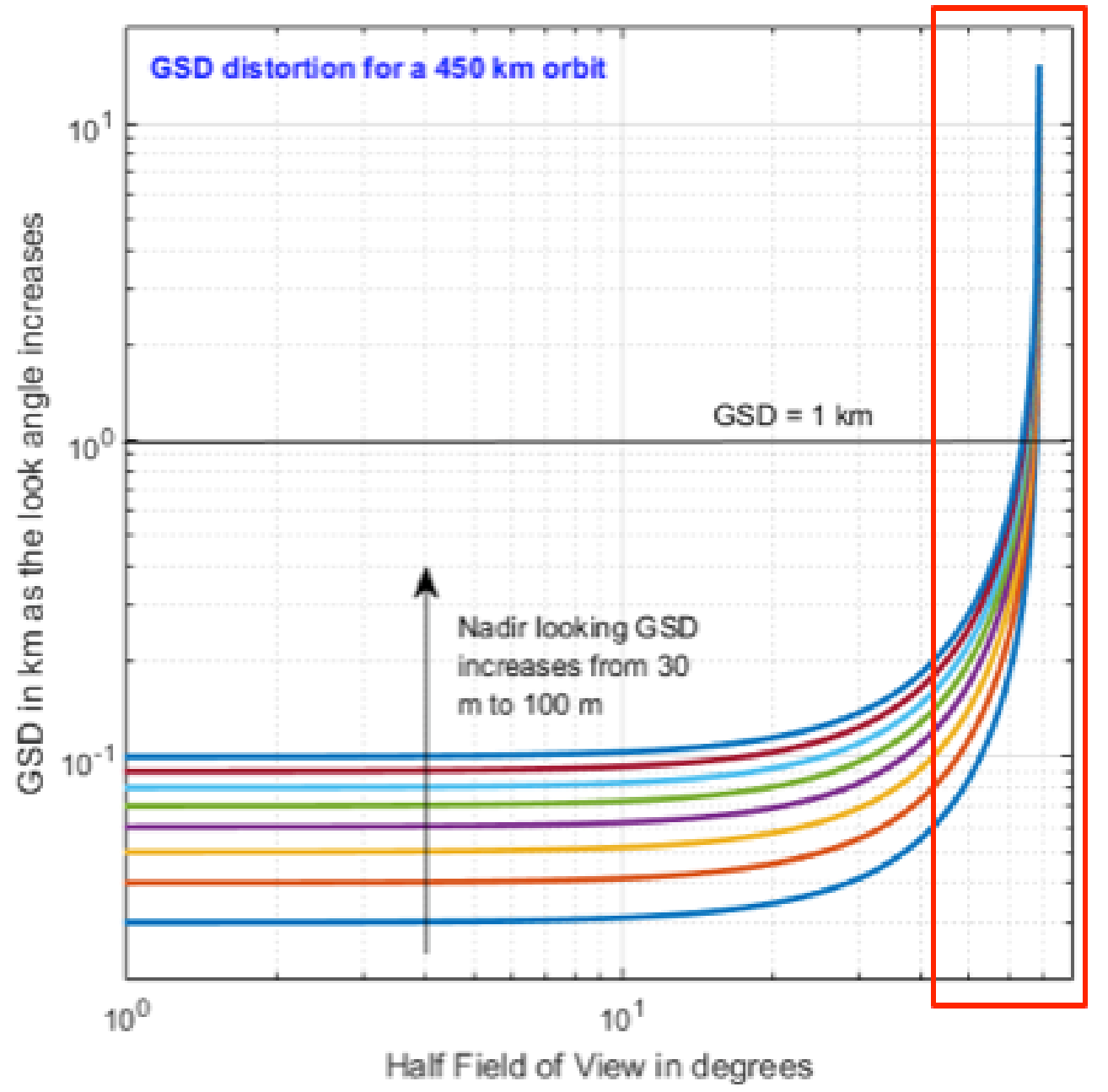




\section{Preliminary Sizing for Streamlining}

\section{Spatial Metric Dependence on Constellation Design Variables:}

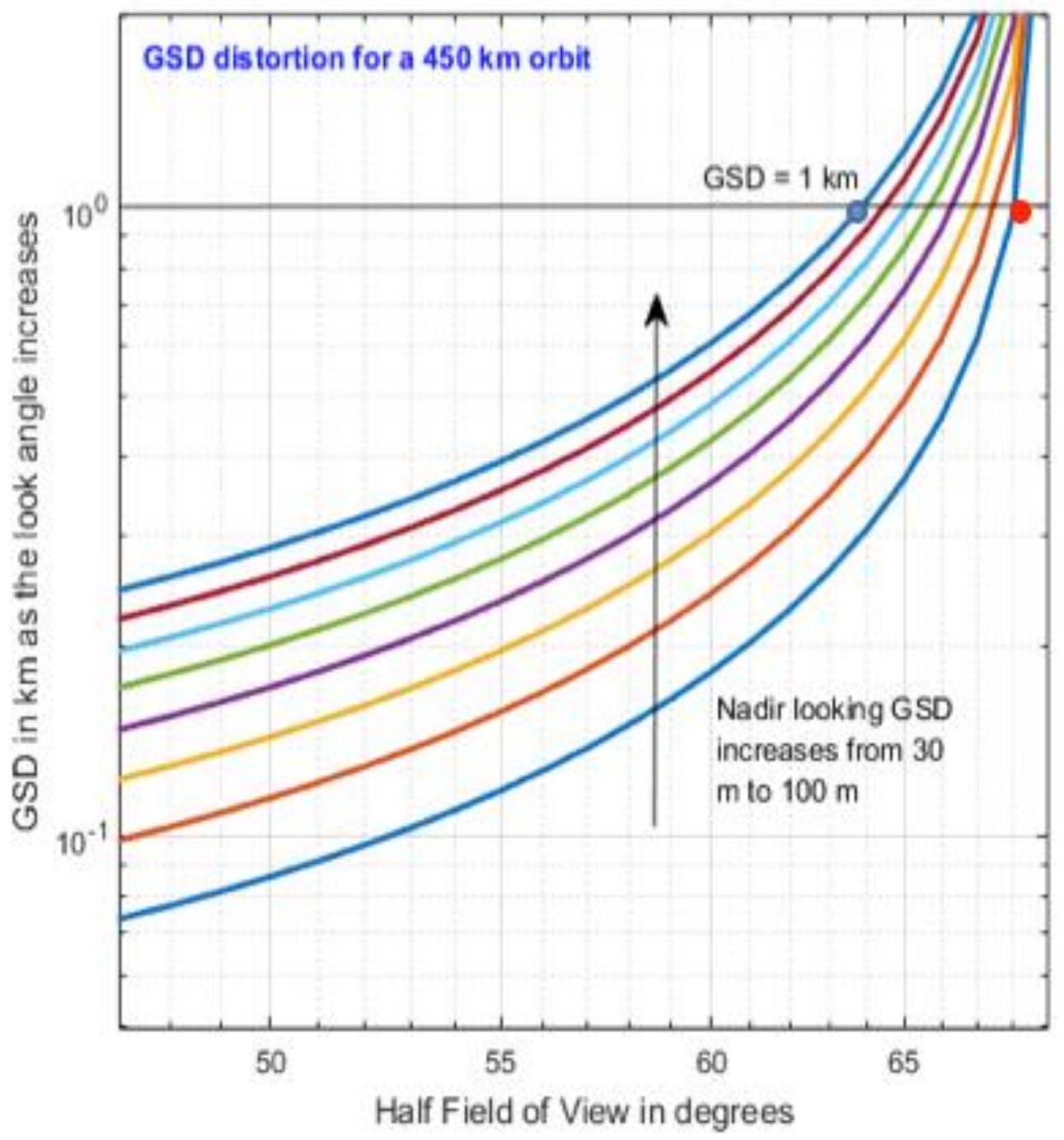

The lower the GSD is at nadir, lower it will be at off-nadir.... 


\section{Preliminary Sizing for Streamlining}

\section{Spatial Metric Dependence on Constellation Design Variables:}

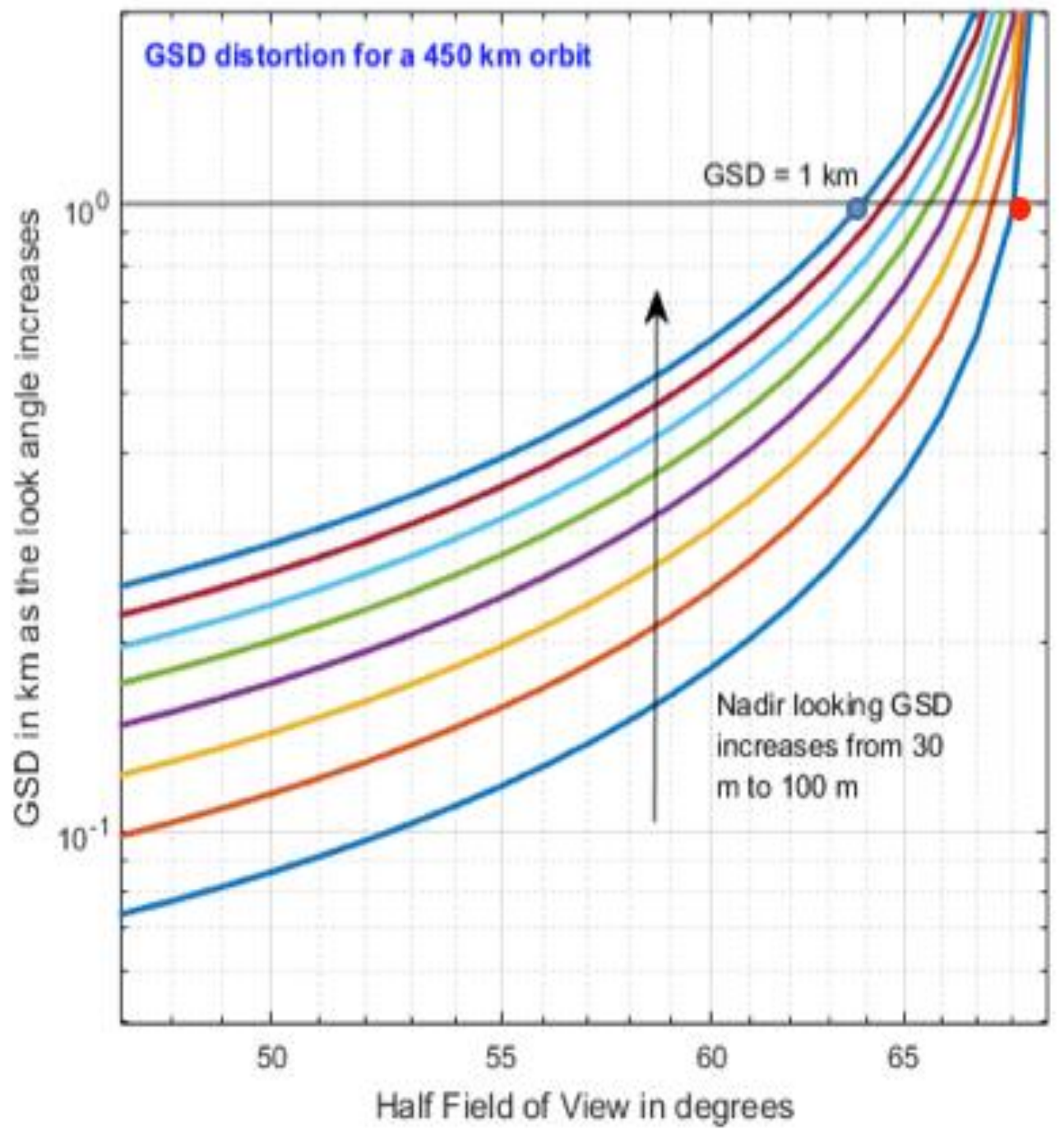

The lower the GSD is at nadir, lower it will be at off-nadir....

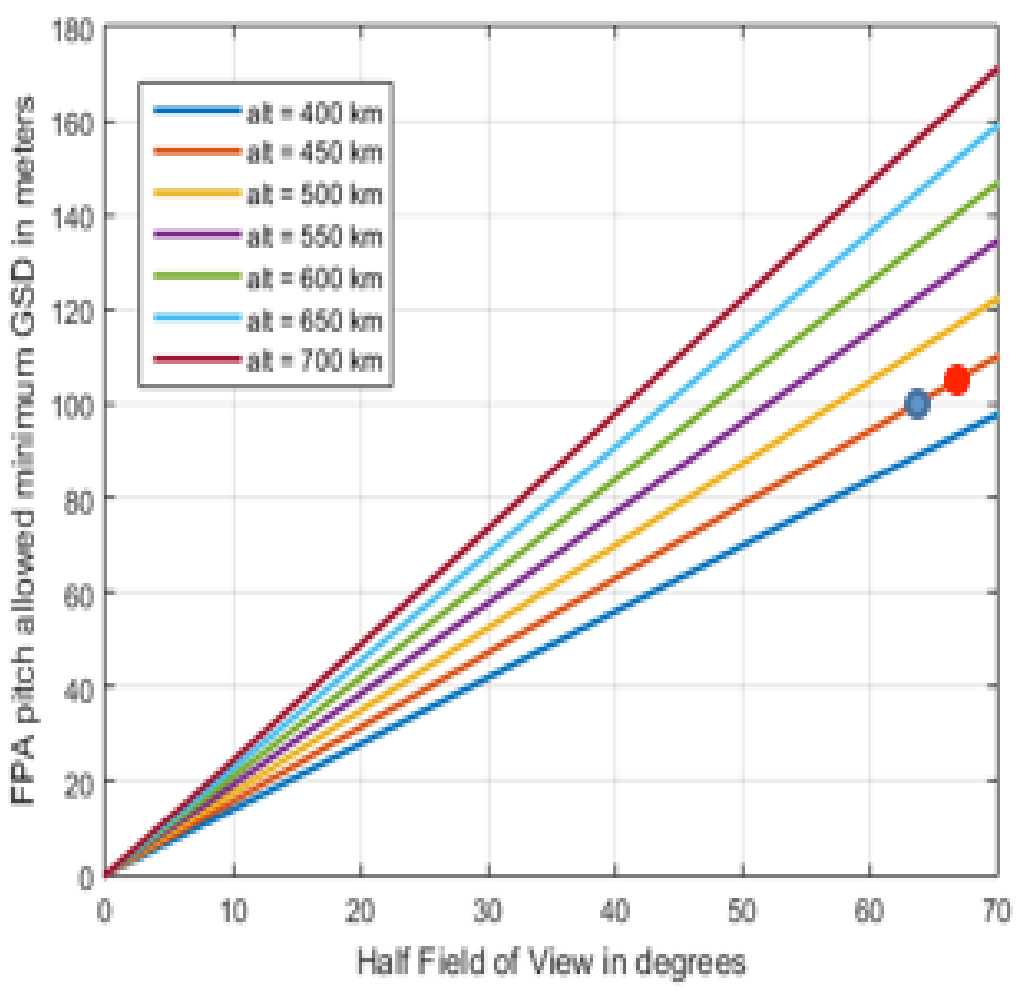

... however, the entire FOV will be more limited due to the physical extent of pixel-delimited imaging. 


\section{Preliminary Sizing for Streamlining}

\section{Temporal Metric Dependence on Constellation Design Variables:}

The maximum and minimum number of satellites can be, very approximately, computed from the swath or an orbital sensor, its altitude and the required revisit time. If the user has specified the range of satellite number desired, those values will override these computations, if they lie outside the computed bounds.

Dependencies can be calculated at run time OR the common ones could be stored and used for streamlining when user variables fall within those values.

\section{Maintenance Predictions:}

- Drag

- J2 caused mean anomaly drift

- RAAN drift relative to orbits is currently minimal due to homogeneous constellations

- No on-off switch for maint yet

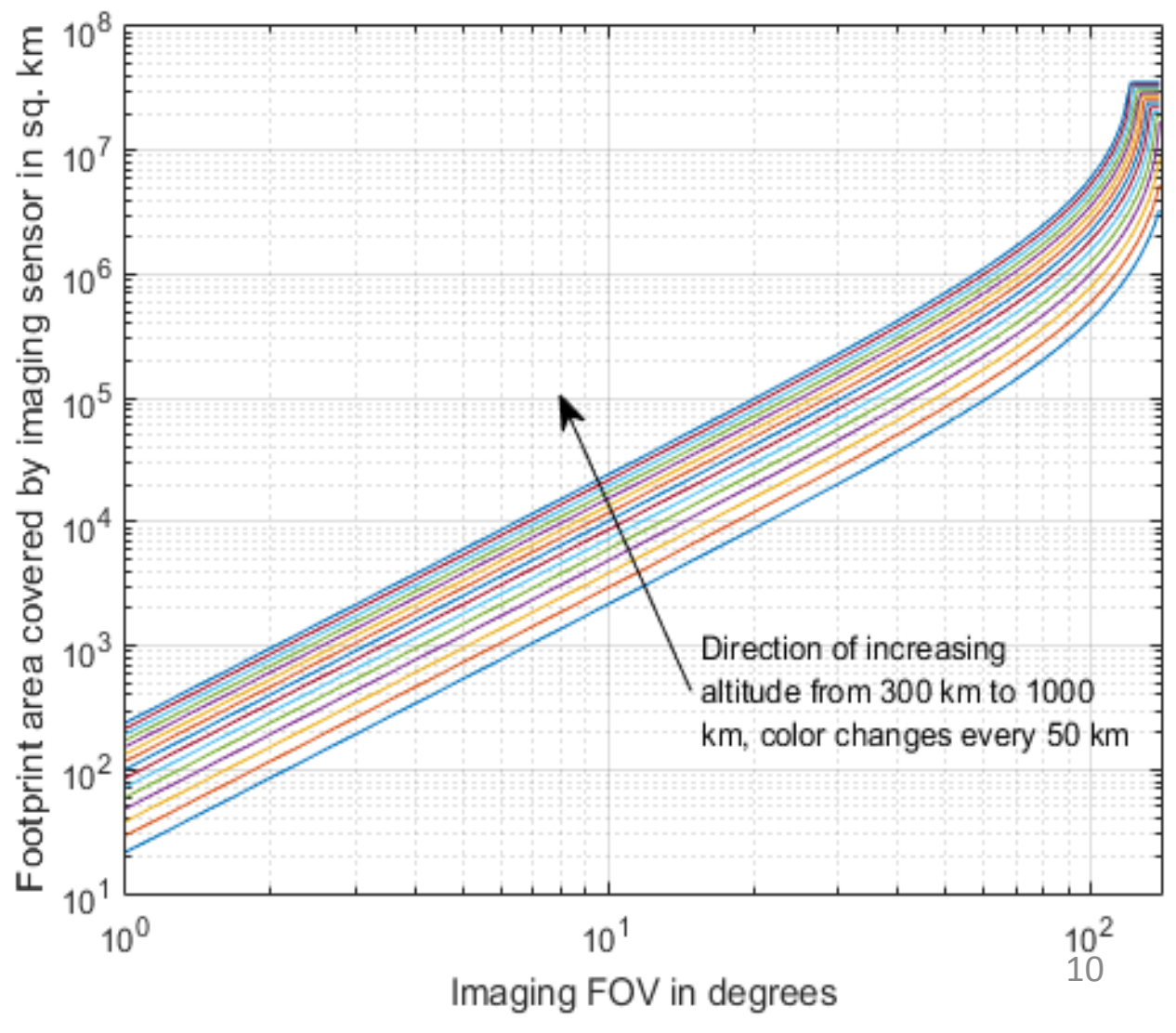




\section{Case Studies - Landsat}

Trades between any pairs or triplets or quadruplets of metrics possible.

Every constellation architecture can be evaluated spatially (and temporally as a time series) using data published by the RM module in gbl.csv and Icl.csv

Landsat Constellation - 1 plane and 8 satellites per plane Maximum Revisit Time

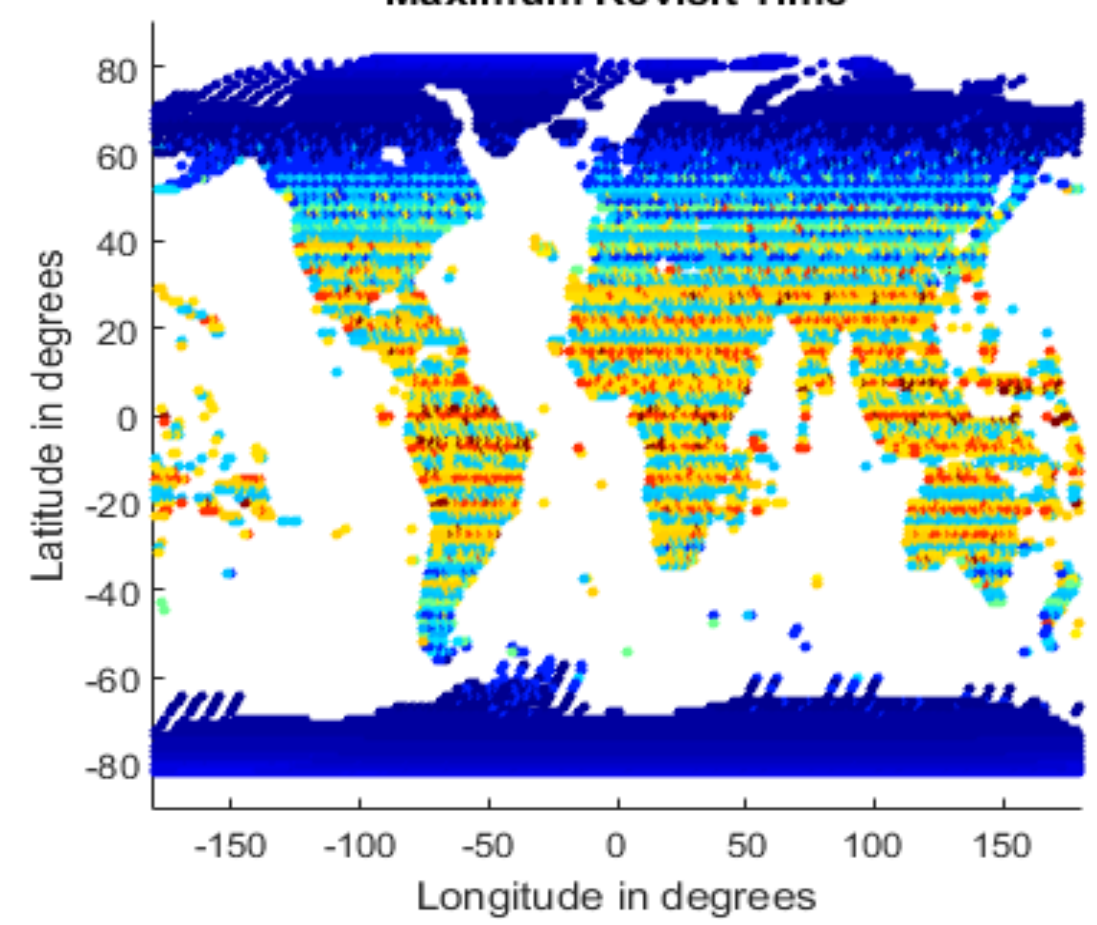

Landsat Constellation - 4 planes and 2 satellites per plane Maximum Revisit Time

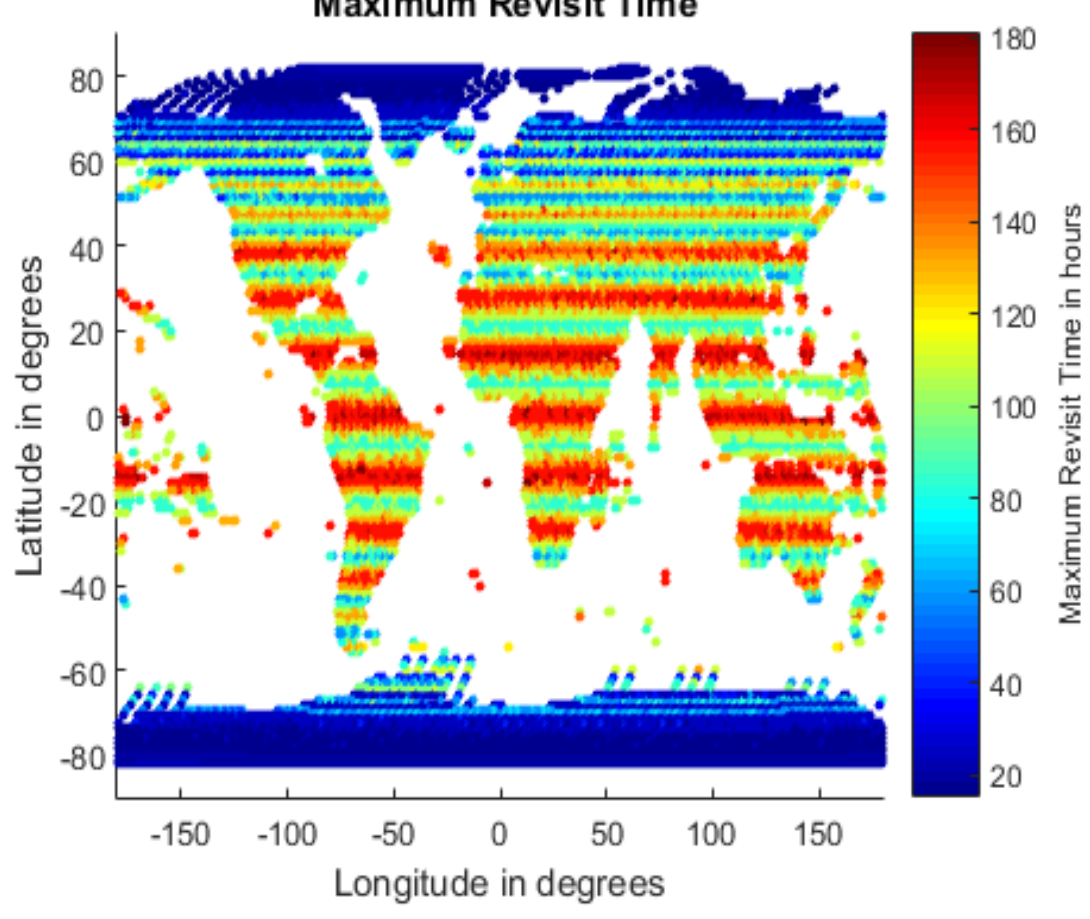

${ }^{* *}$ Figures made with MATLAB for demo purposes. TAT-C's figures will be made with Python 


\section{Case Studies - Landsat}

Trades between any pairs or triplets or quadruplets of metrics possible.

Examples of a few other spatially varying metrics:

Landsat Constellation - 1 plane and 8 satellites per plane Average Revisit Time

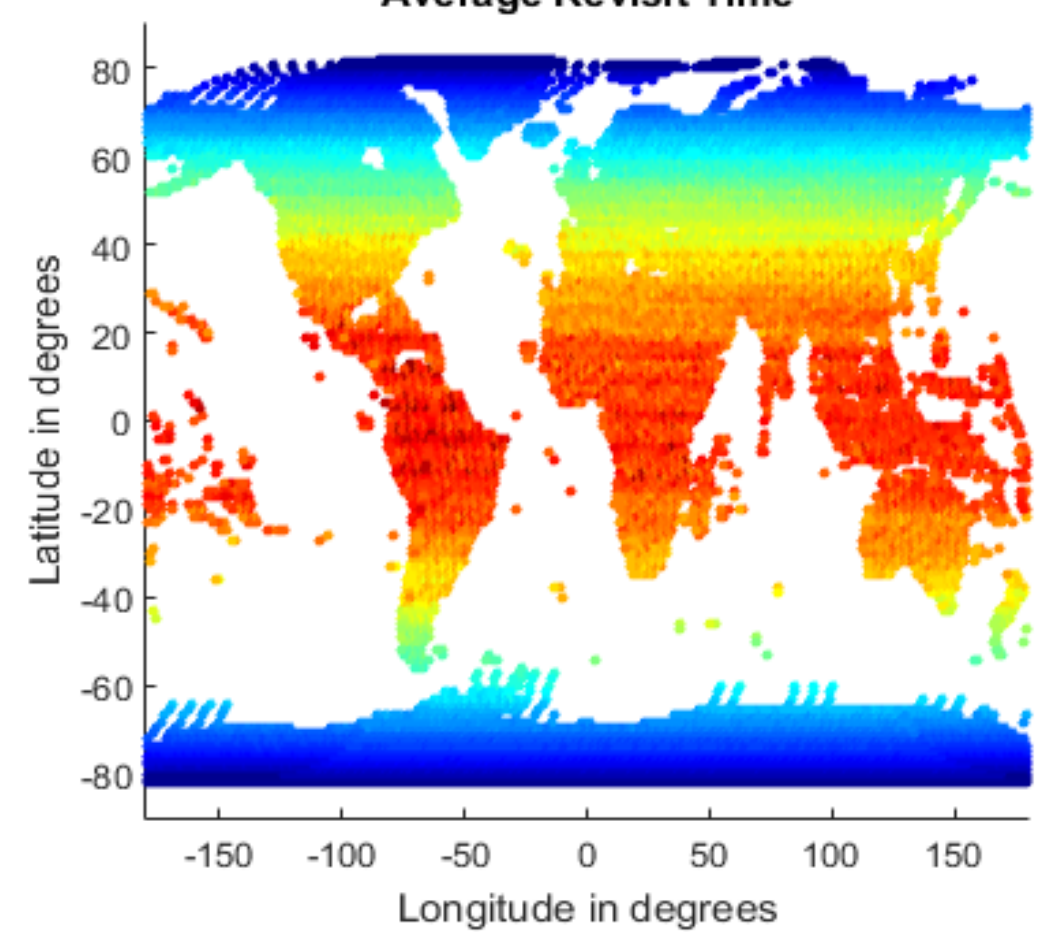

Landsat Constellation - 8 planes and 1 satellite per plane Time to Coverage

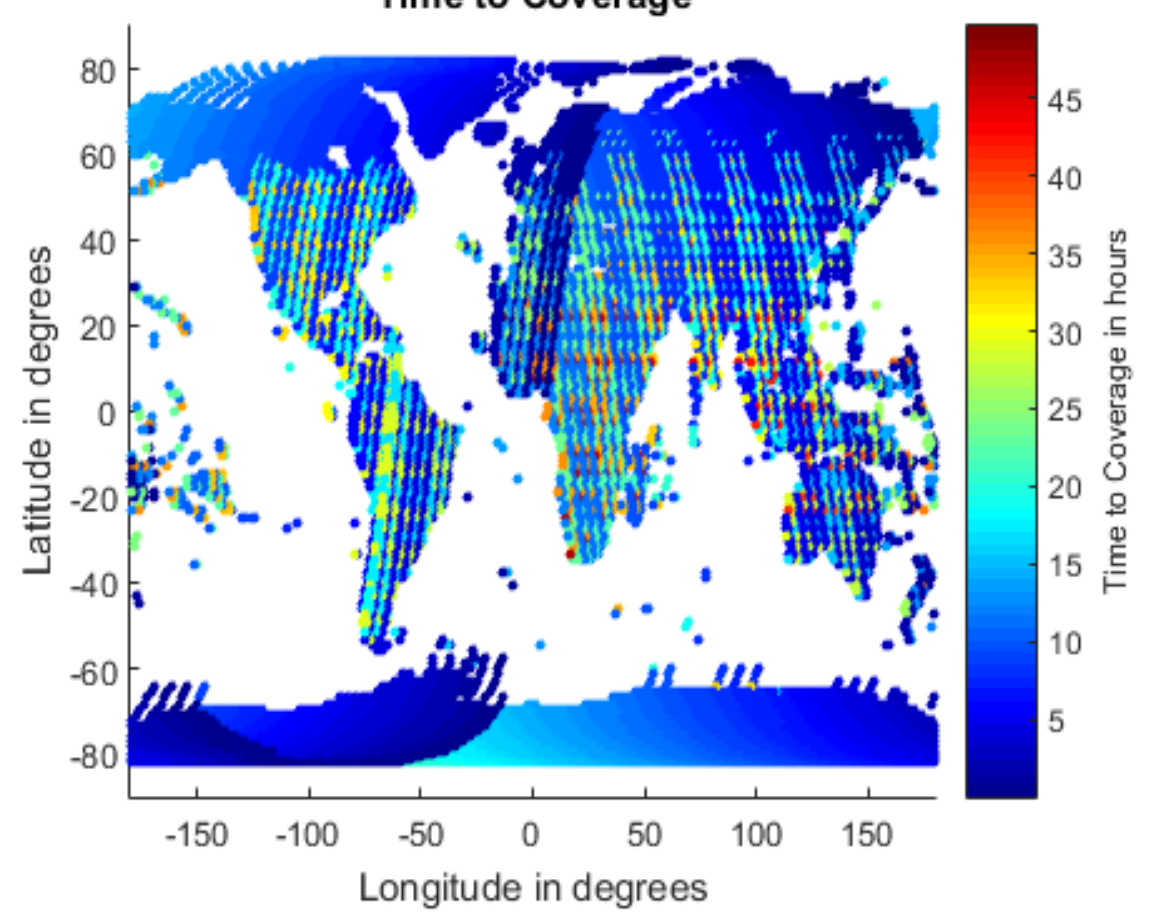

${ }^{* *}$ Figures made with MATLAB for demo purposes. TAT-C's figures will be made with Python 


\section{Case Studies - Wide Angle Radiometer}

130 deg FOV sensor $w / 8-12$ sats in 500-700 km orbit looking within 40 deg parallels $=>228$ uniform Walker constellations and 5 Ad-Hoc constellations (1353 unique orbits). Run time $\mathbf{=} \mathbf{4 0}$ minutes.
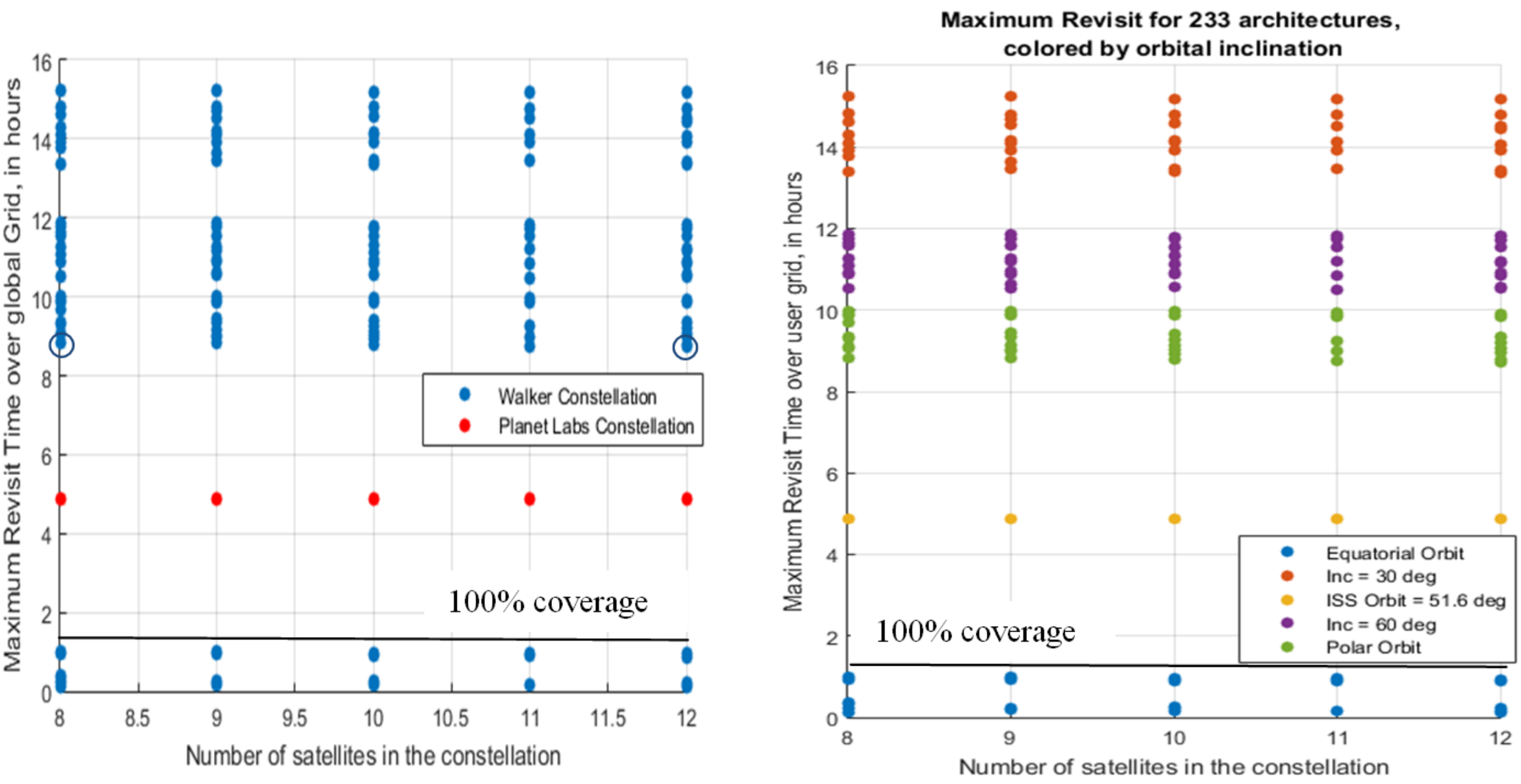


\section{Initial Results Validation}

\section{Validation of Grid Points}

The Area of Interest is discretized into grid points (user provided or OC generated)

The algorithm to place near-equally spaced points was termed the "Helical" algorithm by Schiff and Mailhe [7] as the points look much like what is obtained by peeling an orange in one piece.

Grid size of $10^{\circ}, 5^{\circ}$ and $1^{\circ}$ for a full Earth grid $=>\mathrm{RM} / \mathrm{OC}$ generated 412,1650 and 41252 points respectively.

In comparison, STK generated 410, 1652 and 43424 points respectively.

ED thereby demonstrated less than $1 \%$ of grid spacing error globally.

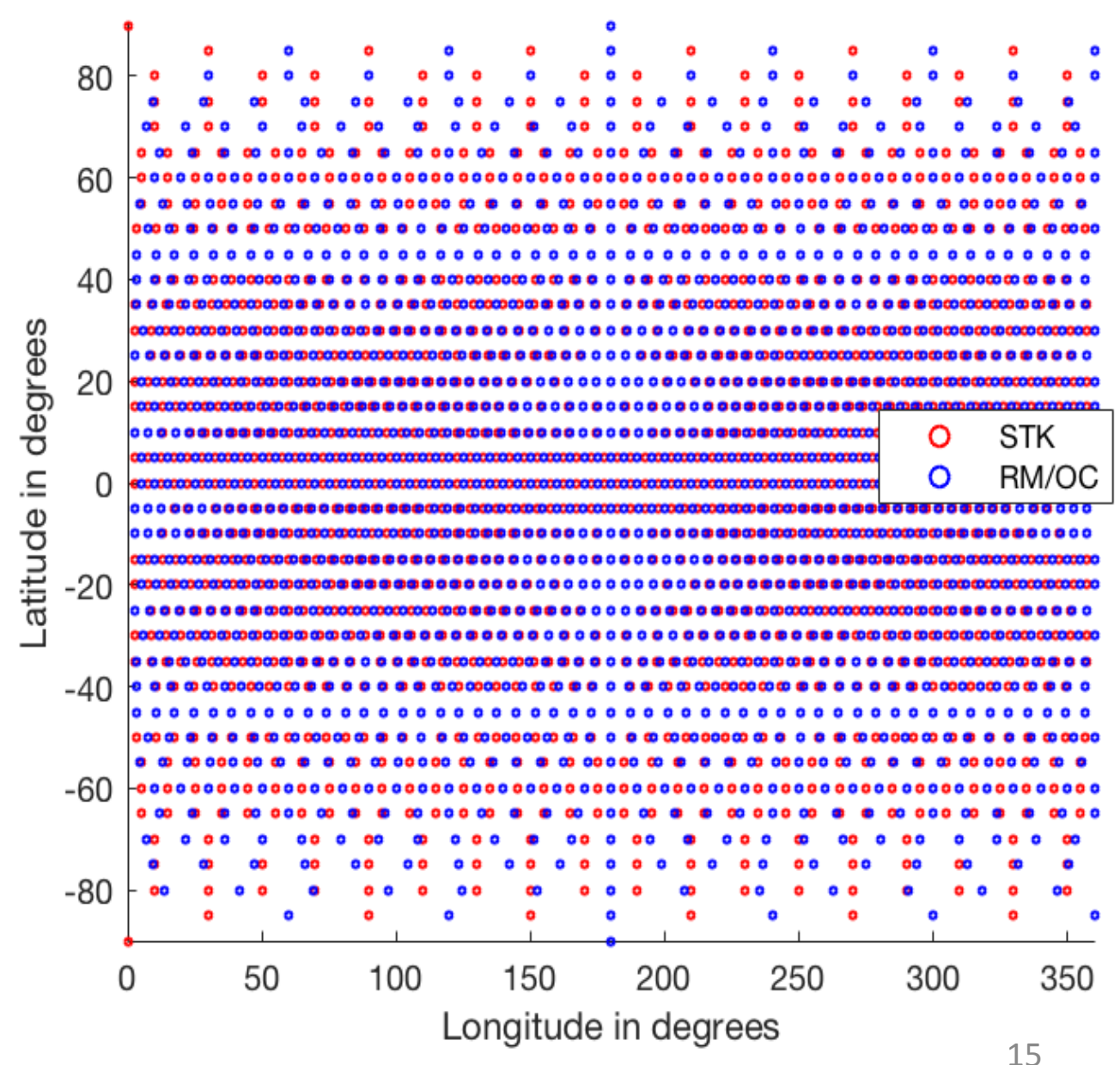




\section{Initial Results Validation}

\section{Validation of Temporal Metrics - Landsat Use Case}

STK+Matlab generated results vs. ED+RM+OC generated results. STK because of its high standing in the orbital mechanics community, utilization in several mission designs and decades of commercial success

Relative shape of the curves, especially in the average revisit time, is similar. Difference could be because of lower fidelity models in OC. $<15$ hrs vs. $10+$ days i.e. 16 times speed.

SECONDARY CHECK: maximum revisit times for WFOV radiometer $=10.4883$ hours by STK vs. 8.83 hours and 8.73 hours by ED, RM, OC. BUT for the single satellite revisit numbers matched up exactly. 1-5 sats $=>10$ mins

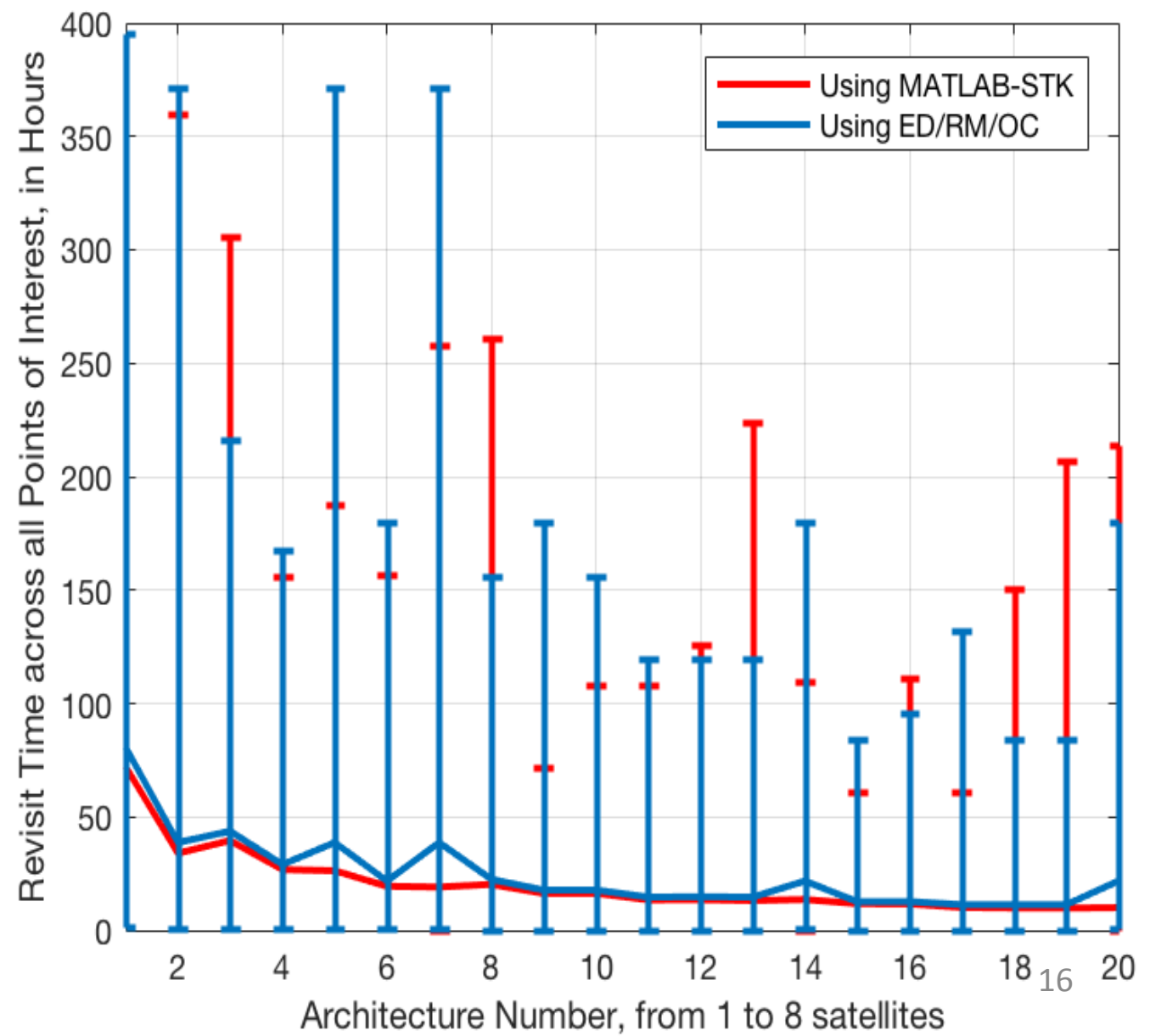




\section{Summary / Future Work}

- Software tools for the pre-phase A design of constellations for Earth Science are essential to understand trade-offs at the concept stage

- TAT-C will facilitate DSM Pre-Phase A investigations and by allowing the users to optimize DSM designs with respect to a-priori science goals [Full tool in a future publication]

- Executive Driver (ED), Orbit and Coverage (OC), Data Reduction and Metric Computation (RM) modules read user inputs and output constraints, generate architectures of constellations, propagate them and evaluate metrics

- Use Cases - Landsat, Wide Angle Radiometer. Results validated against AGI STK

- Future work: Heterogeneous constellations and precession type constellations; Scaling tables within the ED where partial outputs can be processed to inform further tradespace; Concept of operations for non-imaging missions (e.g. occultation). 


\section{Acknowledgements}

Funding: NASA Earth Science Technology Office (ESTO) Advanced Information Systems Technology Program 2014 (2015-2017)

Other Team Members: Matt Holland, Olivier de Weck, Philip Dabney and Veronica Foreman

Other AIAA presentation: Date/Time/Venue 


\title{
Thank you!
}

\author{
Questions?
}

Sreeja.Nag@nasa.gov 